Recurrence in coined quantum walks

This article has been downloaded from IOPscience. Please scroll down to see the full text article.

2009 Phys. Scr. 2009014055

(http://iopscience.iop.org/1402-4896/2009/T135/014055)

The Table of Contents and more related content is available

Download details:

IP Address: 148.6.138.5

The article was downloaded on 05/08/2009 at $14: 38$

Please note that terms and conditions apply. 


\title{
Recurrence in coined quantum walks
}

\author{
T Kiss ${ }^{1}$, L Kecskés ${ }^{1}$, M Štefaňák ${ }^{2}$ and I Jex ${ }^{2}$ \\ ${ }^{1}$ Research Institute for Solid State Physics and Optics, Hungarian Academy of Sciences, Konkoly-Thege \\ M. u. 29-33, H-1121 Budapest, Hungary \\ ${ }^{2}$ Department of Physics, FJFI ČVUT v Praze, Břehová 7, 11519 Praha 1-Staré Město, Czech Republic \\ E-mail: tkiss@szfki.hu
}

Received 8 January 2009

Accepted for publication 12 January 2009

Published 31 July 2009

Online at stacks.iop.org/PhysScr/T135/014055

\begin{abstract}
Recurrence of quantum walks on lattices can be characterized by the generalized Pólya number. Its value reflects the difference between a classical and a quantum system.

The dimension of the lattice is not a unique parameter in the quantum case; both the coin operator and the initial quantum state of the coin influence the recurrence in a nontrivial way. In addition, the definition of the Pólya number involves measurement of the system.

Depending on how measurement is included in the definition, the recurrence properties vary. We show that in the limiting case of frequent, strong measurements, one can approach the classical dynamics. Comparing various cases, we have found numerical indication that our previous definition of the Pólya number provides an upper limit.
\end{abstract}

PACS numbers: 03.67.Ac, 05.40.Fb

\section{Introduction}

Quantum walks provide an important tool to design efficient quantum algorithms [1]. Beyond their possible practical relevance, a fundamental question about quantum walks is the difference between them and their classical counterparts. Of the two major types of quantum walks [2], namely continuous time $[3,4]$ and discrete time [5-7], we will consider here the latter type.

Algorithmic speedup is related to quadratically faster spreading of the probability density during the unitary evolution of the quantum walk with respect to the classical case. One way to quantify the speed of the walk is to examine its hitting time [8-11]. On the other hand, interference among various paths can be destructive as well, resulting in infinite hitting times [10]. Recurrence to the origin can be thought of as a special case of a hitting time.

Recurrence of classical random walks on lattices was first studied by G Pólya [12] who could prove that the return probability to the origin (named after him as the Pólya number) depends solely on the dimensionality of the lattice. For one and two dimensions, the return probability is one, whereas for higher dimensions it is smaller than one, the walks being called recurrent and transient, respectively [13].

One of the fundamental differences between classical and quantum physics is the role of measurement in a physical process. In general, observation of a quantum system unavoidably disturbs it. Nevertheless, the act of measurement is part of every quantum algorithm. When defining recurrence or hitting time, the disturbance of the measurement will change the phenomena depending on how measurements were carried out.

We have defined the Pólya number for quantum walks [14] aiming at minimal disturbance of the unitary evolution. For each measurement, one can take a different member of an ensemble of systems and let it evolve for a different number of time steps. One sequence of such measurement events either contains the origin or does not. The probability of the former case is the Pólya number according to our definition. We have derived various consequences of this definition [15]. Especially, we have shown that in the quantum case not only the dimensionality of the lattice but also the coin operator as well as the initial coin state influence recurrence.

In the present paper, first we give a short review of our definition of the Pólya number and its properties. Next we present possible alternative definitions and evaluate them for various cases. Our results indicate that the original definition [14] gives an upper limit for the possible alternative quantum Pólya numbers.

\section{The quantum Pólya number and its properties}

Let us consider a $d$-dimensional lattice $\mathbb{Z}^{d}$. The quantum walk is defined on the Hilbert space of the corresponding position 
states $\mathcal{H}_{P}=\ell^{2}\left(\mathbb{Z}^{d}\right)=\operatorname{Span}\left\{|\mathbf{m}\rangle \mid \mathbf{m} \in \mathbb{Z}^{d}\right\}$ and the additional coin space $\mathcal{H}_{C}=\operatorname{Span}\left\{\left|\mathbf{e}_{i}\right\rangle \mid i=1, \ldots, c\right\}$, where the possible displacement vectors $\mathbf{e}_{i}$ label the coin states. We use the convention that in each time step the walker is displaced in each spatial dimension; thus all entries in $\mathbf{e}_{i}$ are \pm 1 and $c=2^{d}$.

The evolution in one iteration is governed by the unitary operator

$$
U=S\left(I_{\mathrm{P}} \otimes C\right) .
$$

The coin operator $C$ acts on the coin space, similar to a coin flip, and in our case all of its elements have the same absolute value in its natural representation. $I_{\mathrm{P}}$ is the unit operator on the position space. The step operator reads

$$
S=\sum_{\mathbf{m}, i}\left|\mathbf{m}+\mathbf{e}_{i}\right\rangle\left\langle\mathbf{m}|\otimes| \mathbf{e}_{i}\right\rangle\left\langle\mathbf{e}_{i}\right| .
$$

The initial state is the zero position state (origin), but the initial coin state is arbitrary. The Pólya number, according to our previous definition, as described in the Introduction section, reads

$$
P_{q}=1-\prod_{t=1}^{+\infty}\left(1-p_{0}(t)\right),
$$

where $p_{0}(t)$ is the probability to be at the origin at time $t$. Since the evolution is stopped after each measurement, probabilities at different times are independent in this case. The convergence of the product to zero is mathematically equivalent to the divergence of the sum $\mathcal{S} \equiv \sum_{t=0}^{\infty} p_{0}(t)$.

An explicit evaluation of the probability $p_{0}$ is not necessary for proving the convergence of the sum $\mathcal{S}$; examining how fast it converges to zero is enough. Its asymptotic behavior can be found by analysis of the unitary evolution. In Fourier space, there is a direct method, by applying saddle point approximation, to relate the properties of the eigenvalues of the one-step iteration matrix and the asymptotic decay of $p_{0}(t)$. By using our method, we could determine the Pólya number for various cases.

We could show that in one dimension the Pólya number is always 1 , similar to the classical case. The two-dimensional Grover walk (with a coin operator of real entries, negative for the main diagonal and positive otherwise) has been known to exhibit localization at the origin, leading to recurrence of the walk, except for one initial state, for which there is no localization and the walk is transient. By generalizing the Grover walk, one can explicitly construct a quantum walk in arbitrary dimensions that is recurrent, except for a subset of initial states. Another interesting special case is the two-dimensional Fourier coin (with a coin operator similar to discrete Fourier transformation). In this case, there is no localization, and the walk is still recurrent, except for a two-dimensional subspace of initial states. In section 3, we will compare different definitions of the Pólya number for these special cases.

\section{Alternative definitions for the quantum Pólya number}

Introducing different measurement patterns, we have a great degree of freedom in defining the Pólya number. Let us measure the position of the particle after each step. The projective measurement of a quantum system results in a well-defined but probabilistic position eigenstate. After the first step of the one-dimensional Hadamard coin with the starting state $|0\rangle|R\rangle$, we have

$$
\psi=\frac{1}{\sqrt{2}}|1\rangle|R\rangle+\frac{1}{\sqrt{2}}|-1\rangle|L\rangle
$$

and the state of the system is $\psi=|1\rangle|R\rangle$ or $\psi=|-1\rangle|L\rangle$ with equal probability (here $R$ and $L$ refer to the right and left coin states, respectively). Since the problem is translationally symmetric and also exhibits symmetry for the exchange of left and right, the next step will be similar, only shifted. There is no chance of observing interference between possible paths. In other words, in each step the walker will simply step left or right with $50-50 \%$ probability, the process being equivalent to the classical, symmetric random walk.

In order to deviate from the classical case, a natural possibility is to change the measurement operator. From the point of view of recurrence, it is enough to measure the presence of the particle at the origin. When finding the particle at the origin the evolution stops; otherwise it continues. A related problem has been solved in [16]. They consider quantum walk on a half-line with an absorbing boundary at the origin. In our case the complete line is allowed, but measurement in each step at the origin obstructs interference of the two half-lines. After the first step, we have $\frac{1}{\sqrt{2}}|1\rangle|R\rangle$ on the right half and $\frac{1}{\sqrt{2}}|-1\rangle|L\rangle$ on the left half, evolving separately.

Following [16], one can use a combinatorial approach to find the probability emerging from one half-line and by a generating function approach one can calculate explicitly the recurrence probability. The two half-lines can then be combined, resulting in the total $2 / \pi$ for the Pólya number, proving the transience of the walk.

Less frequent measurement of the position only at the origin leads to a family of quantum Pólya numbers. Depending on whether measurements are carried out regularly (in each $n$th step) or randomly (with equal probability in each step), two sub-cases emerge. We collect results for all these possibilities for the two-dimensional Grover and Fourier coins in figure 1.

\section{Summary}

We have compared various definitions of the quantum Pólya number by varying the measurement frequency. For one dimension, both the classical case and our original definition of the Pólya number indicate recurrence of the walk. Frequent position measurements in the quantum case give back the classical walk. In contrast, frequent measurement of the position only at the origin leads to transience of the walk. For this case we could analytically calculate the Pólya number having the value $2 / \pi$. Comparison of the numerical results for various other definitions suggests that our original definition gives an upper limit for the quantum Pólya number. 
$\Psi_{0}=\frac{1}{2}(1, i, i,-1)$

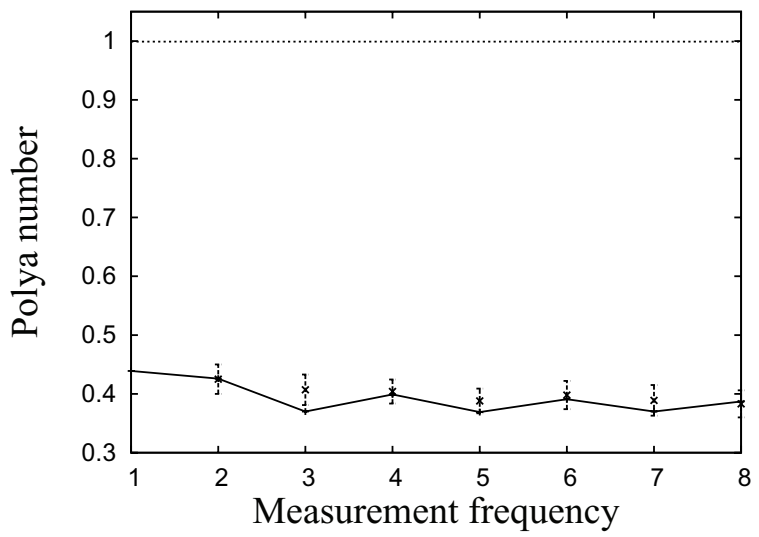

$\Psi_{0}=(1,0,0,0)$

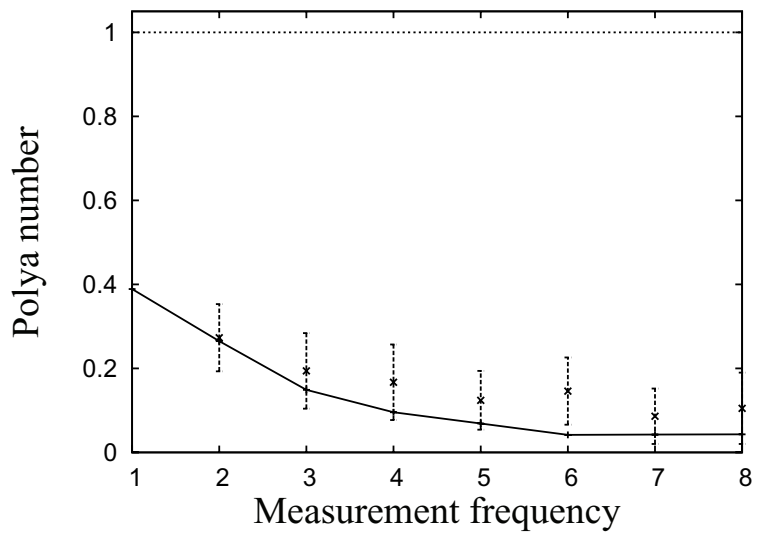

$\Psi_{0}=\frac{1}{2}(1,-1,-1,1)$

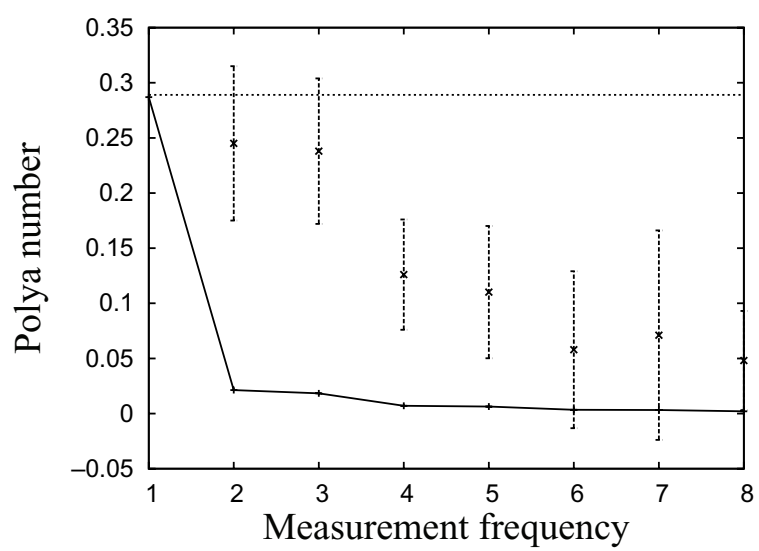

$\Psi_{0}=\frac{1}{2}\left(1, \frac{1-i}{\sqrt{2}}, 1,-\frac{1-i}{\sqrt{2}}\right)$

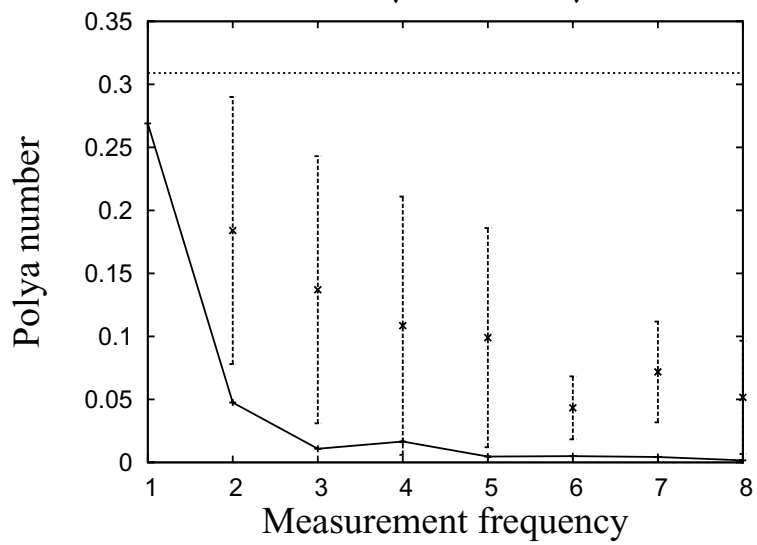

Figure 1. The Pólya number depending on the measurement frequency for various two-dimensional quantum walks. Dotted lines show the original definition of the quantum Pólya number and solid lines show regular measurements; stars with error bars show randomly distributed measurements, each calculated from an ensemble of 100 runs; error bars show standard deviations. The first row corresponds to the Grover coin operator and the second row to the Fourier coin, with the initial coin states $\Psi_{0}$.

\section{Acknowledgments}

Financial support from the MSM (6840770039), the MŠMT (LC 06002), the Czech-Hungarian cooperation project (KONTAKT; CZ-10/2007) and the Hungarian Scientific Research Fund (contract no. T049234) is gratefully acknowledged.

\section{References}

[1] Santha M 2008 5th Theory and Applications of Models of Computation (TAMC08), Xian LNCS 4978 pp 31-46

[2] Aharonov Y, Davidovich L and Zagury N 1993 Phys. Rev. A 481687

[3] Farhi E and Gutmann S 1998 Phys. Rev. A 58915

[4] Childs A, Farhi E and Gutmann S 2002 Quantum Inf. Process 135
[5] Meyer D 1996 J. Stat. Phys. 85551

[6] Meyer D 1996 Phys. Lett. A 223337

[7] Watrous J 2001 J. Comput. Syst. Sci. 62376

[8] Kempe J 2005 Probab. Theory Related Fields 133 215-35

[9] Krovi H and Brun T A 2006 Phys. Rev. A 73032341

[10] Krovi H and Brun T A 2006 Phys. Rev. A 74042334

[11] Magniez F, Nayak A, Richter P C and Santha M 2008 On the hitting times of quantum versus random walks arXiv:0808.0084v1 [quant-ph]

[12] Pólya G 1920 Math. Ann. 84149

[13] Révész P 1990 Random Walk in Random and Non-Random Environments (Singapore: World Scientific)

[14] Štefaňák M, Jex I and Kiss T 2008 Phys. Rev. Lett. 100 020501

[15] Štefaňák M, Kiss T and Jex I 2008 Phys. Rev. A 78 032306

[16] Ambainis A, Bach E, Nayak A, Vishwanath A and Watrous J 2001 Proc. ACM Symp. on Theory of Computation (STOC) pp 37-49 